\title{
Associations of variations in the MRF2/ARID5B gene with susceptibility to type 2 diabetes in the Japanese population
}

\author{
Guoqin Wang ${ }^{1,2,7}$, Masafumi Watanabe ${ }^{1,7}$, Yasushi Imai ${ }^{1,7}$, Kazuo Hara $^{3}$, Ichiro Manabe ${ }^{1}$, Koji Maemura ${ }^{1}$, \\ Momoko Horikoshi ${ }^{3}$, Atsuko Ozeki ${ }^{1}$, Chikako Itoh ${ }^{4}$, Takao Sugiyama ${ }^{5}$, Takashi Kadowaki ${ }^{3}$, \\ Tsutomu Yamazaki ${ }^{6}$ and Ryozo Nagai ${ }^{1}$
}

Modulator recognition factor-2 (Mrf2/AT-rich interaction domain (Arid) $5 b$ ) has been revealed to be involved in pathogenesis of atherosclerosis and adipogenesis. Single-nucleotide polymorphisms (SNPs) in the MRF2/ARID5B gene are associated with coronary artery disease (CAD) and has been proposed as a candidate gene for type 2 diabetes (T2D). The study was aimed to determine whether any of the four MRF2/ARID5B SNPs (rs2893880, rs10740055, rs7087507 and rs10761600) associated with susceptibility to CAD are also associated with T2D, and to determine whether SNP genotype influences the levels of adiponectin and other clinical factors. Association of MRF2/ARID5B SNPs was investigated in 500 diabetic patients from the Department of Metabolic Diseases at the University of Tokyo and 243 hospital-based nondiabetic individuals from the Institute for Adult Disease Asahi Life Foundation Hospital and 500 community-based nondiabetic individuals from the Hiroshima Atomic Bomb Casualty Council Health Management Center. Associations of haplotypes of these SNP with levels of adiponectin and other clinical factors were evaluated when the data was available. We found rs2893880C, rs $10740055 \mathrm{~A}$, rs7087507A and rs 10761600T were increasingly associated with T2D in terms of allele/genotype frequencies of each SNP and their haplotype combinations. Individuals with haplotype CAAT indicated an 1.86 times higher prevalence of diabetes compared with individuals with GCGA (OR 1.86 (95\% confidence interval (CI) 1.43-2.41)). Furthermore, CAAT significantly associated with adiponectin levels and other clinical factors. In conclusion, polymorphisms on the MRF2/ARID5B gene were associated with susceptibility to T2D as well as adiponectin and other clinical factors, which was in a completely concordant way with their associations with CAD.

Journal of Human Genetics (2012) 57, 727-733; doi:10.1038/jhg.2012.101; published online 13 September 2012

Keywords: Adiponectin; ARID5B; coronary artery disease; diabetes; genetic risk factors; MRF2; polymorphism

\section{INTRODUCTION}

Although multiple environmental factors as well as different genes indentified by single nucleotide polymorphisms (SNPs) and genomewide association studies were thought to contribute to type 2 diabetes (T2D), ${ }^{1-4}$ its pathophysiological basis remains to be elucidated. ${ }^{5}$ Modulator recognition factor-2 (MRF2) is a member of the AT-rich interaction domain (ARID) family of transcription factors (also known as ARID5B or Desrt), binding with high affinity to the target sequence AATA $(\mathrm{C} / \mathrm{T}){ }^{6,7}$ We previously reported the full length of MRF2/ARID5B and demonstrated its regulatory role for the phenotypic change of smooth muscle cells, which was considered as a crucial process for the pathogenesis of atherosclerosis. ${ }^{8}$ On the other hand, targeted disruption of the Mrf2/Arid5b gene in mice has been reported to cause growth retardation and significant reductions in lipid accumulation and weight gain in postnatal and adult life. ${ }^{9-11}$ Recent in vitro studies supported these findings and provided evidence of its role on adipogenesis. ${ }^{12-14}$ All these are considered to be critical aspects in metabolic syndrome and T2D. We therefore hypothesized that genetic variations in the MRF2/ARID5B gene may predispose humans to coronary artery disease (CAD) and T2D. In our previous study, we confirmed the association of MRF2/ARID5B polymorphisms with susceptibility to CAD. ${ }^{15}$ This study is to investigate whether or not these variations are also consistently associated with the presence of $\mathrm{T} 2 \mathrm{D}$ and relevant clinical factors.

\footnotetext{
${ }^{1}$ Department of Cardiovascular Medicine, Graduate School of Medicine, the University of Tokyo, Tokyo, Japan; ${ }^{2}$ Kitasato Clinical Research Center, School of Medicine, Kitasato University, Sagamihara, Japan; ${ }^{3}$ Department of Diabetes and Metabolism, Graduate School of Medicine, the University of Tokyo, Tokyo, Japan; ${ }^{4}$ Hiroshima Atomic Bomb Casualty Council Health Management Center, Hiroshima, Japan; ${ }^{5}$ nstitute for Adult Diseases, Asahi Life Foundation, Tokyo, Japan and ${ }^{6}$ Department of Clinical Epidemiology and Systems, Graduate School of Medicine, the University of Tokyo, Tokyo, Japan

${ }^{7}$ These authors contributed equally to this work.

Correspondence: Dr M Watanabe or Dr G Wang, Department of Cardiovascular Medicine, The University of Tokyo Hospital, 7-3-1 Hongo, Bunkyo-ku, Tokyo 113-8655, Japan. E-mail: masafumi-tky@umin.ac.jp or wangguoqin@kitasato-crc.org

Received 16 January 2012; revised 5 July 2012; accepted 17 July 2012; published online 13 September 2012
} 


\section{MATERIALS AND METHODS}

Individuals and materials

This case-control study included 500 T2D patients ( 317 men and 183 women, $62.7 \pm 10.1$ years old, body mass index (BMI) $24.6 \pm 4.2$ ) and 743 nondiabetic individuals consisting 243 hospital-based individuals in control group 1 (130 men and 113 women, $53.5 \pm 11.1$ years old, BMI 22.9 \pm 4.1$)$ and 500 community-based individuals in control group 2 (235 men and 265 women, $68.5 \pm 6.9$ years old, BMI $24.5 \pm 4.0$ ). The T2D patients were randomly selected from the outpatient clinic of the Department of Metabolic Diseases at the University of Tokyo (Tokyo, Japan), as previously described. ${ }^{16}$ T2D was diagnosed according to the criteria of the World Health Organization. The individuals in control group 1 were recruited in the year 2000 from the monthly follow-up or health check-up program at the outpatient clinic of the Institute for Adult Disease Asahi Life Foundation Hospital based on $>20$ years old and without definitely diagnosed type 1 or 2 diabetes or CAD. This group was included to give differences between patients and the hospital-based healthy subjects. The individuals in control group 2 were recruited from those undergoing routine health check-ups at the Hiroshima Atomic Bomb Casualty Council Health Management Center (Hiroshima, Japan) on the basis of: (1) $>50$ years of old, (2) with hemoglobin A1c ( $\mathrm{HbAlc}$ ) values $<6.2 \%$ and (3) having no family histories of T2D in the first- or second-degree relatives. ${ }^{16,17}$ The above criteria were chosen to detect differences between patients and the community-based health subjects with the aim of enhancing the power of the analyses. All participants were of full Japanese ethnicity. The study was approved by the institutional ethics committee of the University of Tokyo, and written informed consent was obtained from each participant.

Baseline information was collected at the point of enrollment. BMI was assessed with the subject wearing a scrub suit and no shoes. Blood samples were drawn in the fasting state, in which fasting plasma glucose (FPG), insulin and HbAlc levels were measured. HbAlc was given in NGSP (National Glycohemoglobin Standardization Program\%), which is reported to be reasonably estimated by the equation of JDS (Japan Diabetes Society\%) $+0.4 \%{ }^{18}$ Plasma adiponetin levels were measured using a ELISA kit from Fujirebio Inc. (Tokyo, Japan), which has been shown to be able to measure the proportion of the high molecular weight form of adiponectin ${ }^{19}$. As reported previously, a $100-\mu \mathrm{l}$ volume of diluted serum and standard samples was applied to a 96-well microtiter plate coated with mouse antiadiponectin monoclonal antibody. The plate was incubated for an hour and washed and incubated with the same mouse monoclonal antibody labeled with horseradish peroxidase, then, the plate was washed and incubated with tetramethylbenzidine reagent. To stop the reaction, $0.36 \mathrm{~N}$ sulfuric acid solution was added, and the absorbance at $450 \mathrm{~nm}$ was measured. ${ }^{16}$ Intraand inter-assay coefficients of variation of the kit were reported to be below 3.0 and $5.1 \%$, respectively. ${ }^{19}$ Insulin resistance was assessed by homeostasis model assessment (HOMA). Insulin resistance index indicates FPG $\left(\mathrm{mmoll}^{-1}\right) \times$ fasting insulin $\left(\mu \mathrm{U} \mathrm{ml}^{-1}\right) / 22.5^{20}$

\section{Genomic DNA extraction and genotyping of SNPs used in the association study}

Venous blood samples were collected in tubes containing $\mathrm{Na}_{2} \mathrm{EDTA}$ and applied to genomic DNA extracting columns (Genomix DNA Extraction Kit, Talent, Italy) according to the manufacturer's protocol. SNPs were genotyped using a MassARRAY system as described elsewhere. ${ }^{21}$ In brief, primer extension products were analyzed by chip-based matrix-assisted laser desorption/ ionization time-of-flight mass spectrometry following PCR amplification under the standard conditions. The results based on twice consistent independent genotyping, and ambiguous base callings were eliminated from further analysis.

The selection of SNPs of the MRF2/ARID5B gene was based on our previous findings of 11 intronic SNPs, ${ }^{15}$ which covered the entire region of the gene and were selected from the JSNP (Japan Single Nucleotide Polymorphisms) database (http://snp.ims.u-tokyo.ac.jp) or the NCBI (National Center for Biotechnology Information) GeneBank database (http://www.ncbi.nlm. nih.gov/SNP). As described previously, four nearby SNPs (rs2893880, rs10740055, rs7087507 and rs10761600) covering the region of exon 3 and intron 3 of the MRF2/ARID5B gene showed strong linkage disequilibrium (LD) and was assumed to constitute one haplotype block (Figure 1). These variants were associated with CAD and were therefore investigated for their associations with T2D in this study. In addition, another four SNPs beyond this block (Figure 1) were further genotyped to investigate whether a broader area exists that may confer risks to diabetes. Three were investigated in our previous study (rs7901348, rs2278308 and rs12357548) with one (rs10821944) included additionally.

\section{Statistical analyses}

Continuous parameters were presented as mean \pm s.d. or median (25th and 75th percentiles) and were compared using the Student's independent $t$ test, Mann-Whitney $U$ test or Kruskal-Wallis test, as appropriate. Categorical data were given as proportions of all the samples and were compared using the Pearson's chi-square $\left(\chi^{2}\right)$ test. We planned a study with 1.5 controls per case. Based on our previous findings, to observe a $10 \%$ difference in allele or genotype frequencies between the two groups, with a power of 0.8 and at an alpha level of 0.05 , conservatively, 342 diabetic patients and 513 control patients were necessary. For an association to be considered significant, it had to involve the same risk allele as that reported in our previous study. We took potential overestimation into account by applying Bonferroni correction of the number of SNPs when evaluating the most statistically significant values among those tested under different models, because the multiple-comparison nature of the tests lead to higher false-positive rates.

LD coefficients $\left(\mathrm{D}^{\prime}\right)$ of each pair of SNPs were estimated via the maximum likelihood from the two locus genotype data. Haplotypes were estimated by the expectation maximuzation algorithm under the assumption of Hardy Weinberg equilibrium. ${ }^{16}$ Haplotype blocks was defined using the open source software Haploview based on the CI definition developed by Gabrieal et al.,22 where $\mathrm{D}^{\prime}>0.8$ indicates strong $\mathrm{LD}$, and the lower and upper CIs minima for strong LD were set as 0.60 and 0.98 , respectively, in this study. For the estimation of haplotype frequencies, we selected one of the SNPs as a tagging SNP from every set of SNPs with $\mathrm{D}^{\prime}>0.80 .{ }^{15,23}$ All haplotypes were jointly tested for association with disease status by performing a $2 \times n \chi^{2}$ test of independence in a permutation procedure, where ' $n$ ' indicates the number of haplotypes with a frequency $>0.5$. The odds ratios (ORs) and $95 \% \mathrm{CI}$, with adjustments for age, gender and BMI, were calculated by multivariable logistic regression analysis. The statistical analyses were performed using SPSS, version 17.0, software (SPSS Inc., Chicago, IL, USA). LD and haplotype analysis were confirmed with SNPAlyze v5.1 standard software (Dynacom, Yokohama, Japan). A two-tailed value of $P<0.05$ was considered to be significant unless otherwise indicated.

\section{RESULTS}

Baseline characteristics of the diabetic and nondiabetic individuals All SNPs satisfied the Hardy-Weinberg equilibrium, and each of the minor allele frequencies was $>5 \%$. Call rate of each SNP defined by successful typing number divided by total typing number was $>98 \%$. The baseline characteristics of the diabetic patients and the control individuals are shown in Table 1. The combined control group was formed by combining control groups 1 and 2. Control group 1 included higher portion of male, younger individuals, lower BMI and higher $\mathrm{HbAlc}$ than control group 2. Differences between the diabetic and each control group or the combined control group were observed for gender, age, HbAlc, FPG, the levels of adiponectin and other clinical factors.

\section{Associations between MRF2/ARID5B SNPs and susceptibility to T2D}

The allele and genotypic frequencies are depicted in Table 2. The C-allele for rs2893880, A-allele for rs10740055, A-allele for rs7087507 and T-allele for rs10761600 of the T2D patients were more prominent than each of control groups either for the allele frequencies or 


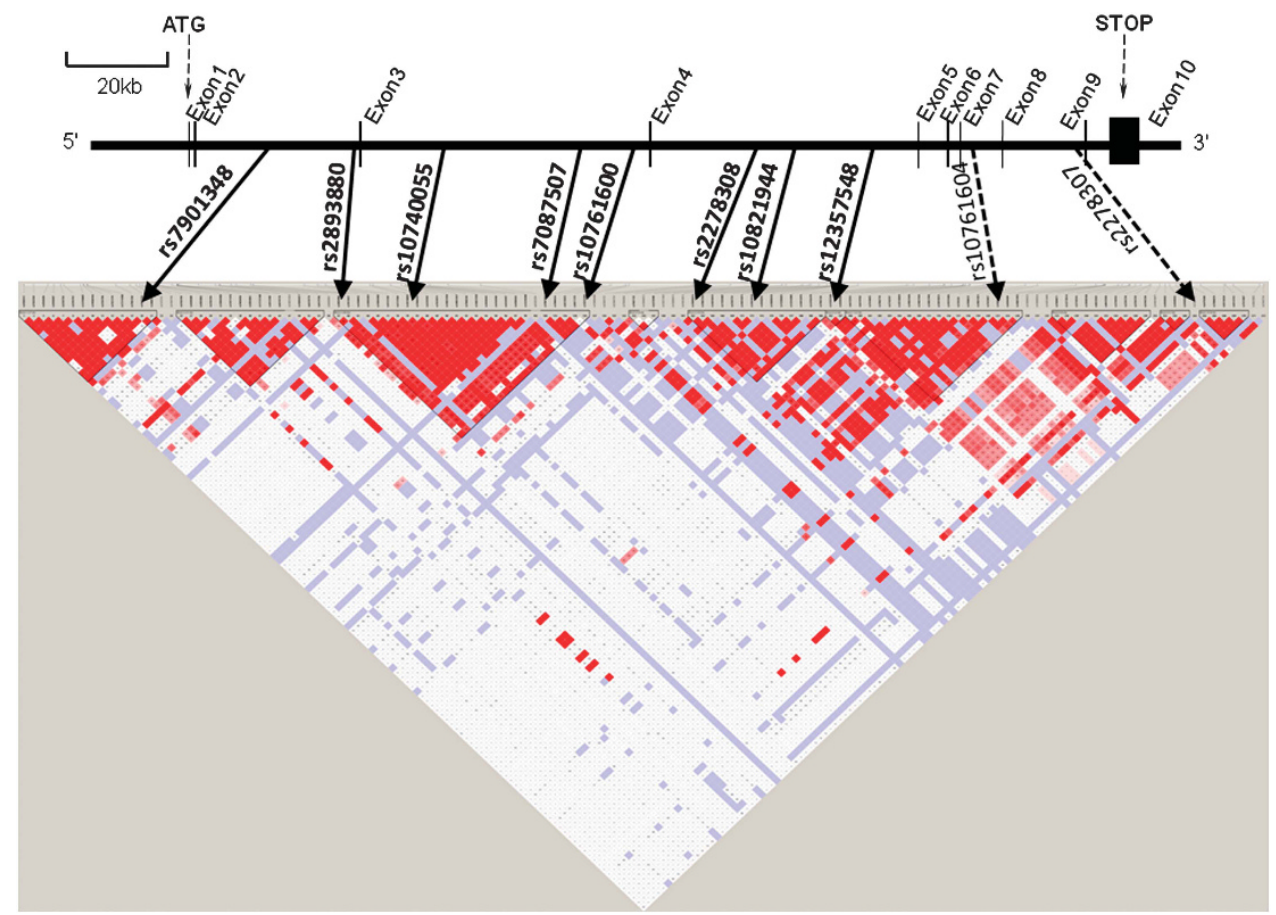

Figure 1 Gene structure and haplotype blocks of MRF2/ARID5B (modulator recognition factor-2/AT-rich interaction domain) single nucleotide polymorphisms (SNPs). Exons differing from $<100 \mathrm{bp}$ to $>1000 \mathrm{bp}$ are indicated by lines or closed boxes. Linkage disequilibrium (LD) structure and haplotype blocks of the MRF2/ARID5B gene were estimated using the Japanese data from the HapMap database. The pairwise LD between SNPs and $D^{\prime}$ are indicated in different colors with red showing strong LD. Haplotype blocks were represented with red triangles with solid black lines. Locations of SNPs investigated in this study were indicated with arrows of solid lines. Two SNPs with arrows of dash lines were investigated previously.

Table 1 Clinical characteristics of the study individuals

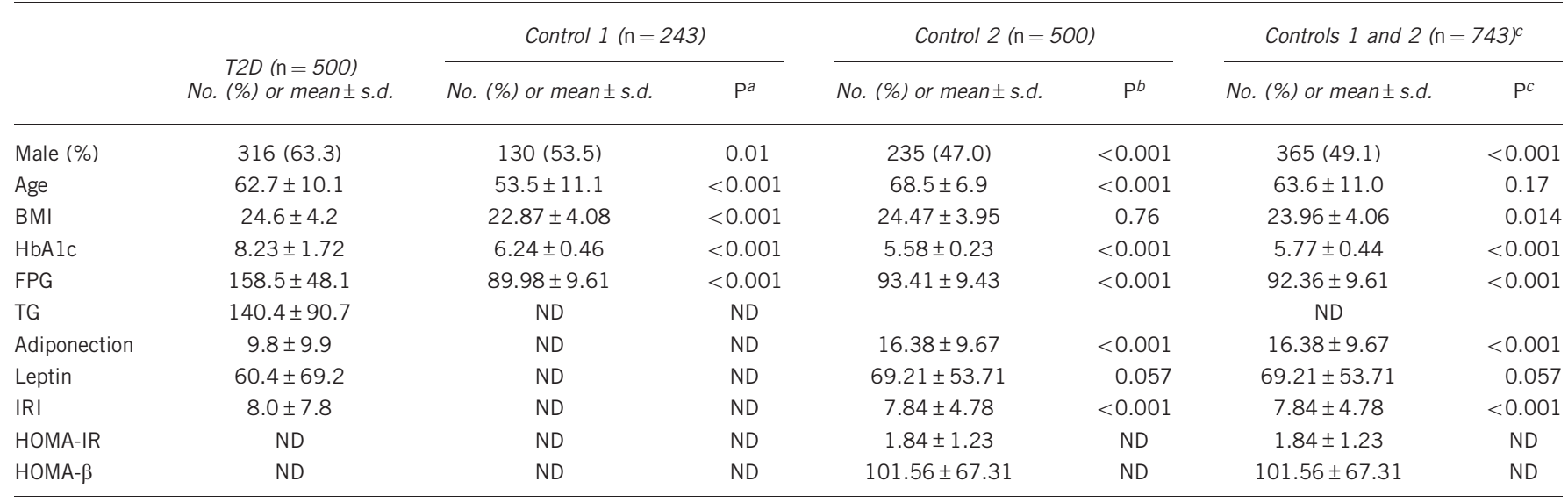

Abbreviations: BMI, body mass index; FPG, fasting plasma glucose; HbAlc, hemoglobin Alc; HOMA-IR, homeostasis model assessment of insulin resistance; IRI, insulin resistance index; ND, not determined; T2D, type 2 diabetes.

asignificance indicates control 1 vs T2D.

bSignificance indicates control 2 vs T2D.

"Significance indicates the combined control group (controls 1 and 2), which are formed by combining control groups 1 and 2, vs T2D.

Continuous parameters were described as mean $\pm \mathrm{s}$.d; differences of age and BMI were compared using Student's $t$ test, and others were tested by Mann-Whitney $U$ test.Gender was given as number (proportions of the entire sample) and tested by chi-square test.

genotypic frequencies. No difference was observed between the two control groups.

Table 3 presents the results by applying a multivariable logistic regression, which gives the effect of each genotype by adjusting for age, gender and BMI. The results from the two control groups were concordant with each other. For rs2893880, compared with codominant-type GG, CC seemed to contribute higher risk to the presence of
T2D (OR 1.52 (95\% CI 1.04-2.23), $P=0.032$ ); for rs7087507, individuals of carrying GG or a single G (recessive model) seemed to decrease risk of presenting T2D than AA (OR 0.71 (95\% CI 0.56$0.90), P=0.005)$. With respect to rs 10740055 , codominant-type AA increased risk of T2D as compared with CC (OR 1.65 (95\% CI 1.20$2.26), P=0.0021)$, and carrying $\mathrm{CC}$ or a single $\mathrm{C}$ (recessive model) prevent individuals from presenting T2D than AA (OR 0.64 (95\% CI 
Table 2 Allele and genotypic frequencies of MRF2/ARID5B SNPs

\begin{tabular}{|c|c|c|c|c|c|c|c|}
\hline & \multirow{2}{*}{$\begin{array}{l}\text { T2D }(\mathrm{n}=500) \\
\text { Frequency }(\%)\end{array}$} & \multicolumn{2}{|c|}{ Control $1(n=243)$} & \multicolumn{2}{|c|}{ Control $2(n=500)$} & \multicolumn{2}{|c|}{ Controls 1 and $2(n=743)$} \\
\hline & & Frequency (\%) & $\mathrm{P}^{a}$ & Frequency (\%) & $\mathrm{P}^{b}$ & Frequency (\%) & $\mathrm{P}^{c}$ \\
\hline \multicolumn{8}{|c|}{ rs2893880 } \\
\hline GG & $52(10.6)$ & $39(16.1)$ & 0.087 & $65(13.1)$ & 0.072 & $104(14.1)$ & 0.043 \\
\hline$C G$ & $202(41.2)$ & $100(41.1)$ & & $226(45.8)$ & & $326(44.2)$ & \\
\hline $\mathrm{CC}$ & $236(48.2)$ & $104(42.8)$ & & $203(41.1)$ & & $307(41.7)$ & \\
\hline G & $306(31.2)$ & $178(36.6)$ & 0.038 & $356(36.0)$ & 0.023 & $534(36.2)$ & 0.011 \\
\hline C & $674(68.8)$ & $308(63.4)$ & & $632(64.0)$ & & $940(63.8)$ & \\
\hline \multicolumn{8}{|c|}{ rs 10740055} \\
\hline $\mathrm{CC}$ & $114(23.3)$ & $70(28.8)$ & 0.045 & $136(27.5)$ & 0.006 & 206 (27.9) & 0.002 \\
\hline$A C$ & $211(43.0)$ & $112(46.1)$ & & $237(48.0)$ & & $349(47.4)$ & \\
\hline$A A$ & $165(33.7)$ & $61(25.1)$ & & $121(24.5)$ & & $182(24.7)$ & \\
\hline $\mathrm{C}$ & $439(44.7)$ & 252 (51.9) & 0.011 & $509(51.5)$ & 0.0024 & $761(51.6)$ & 0.00091 \\
\hline A & $541(55.3)$ & $234(48.1)$ & & $479(48.5)$ & & $713(48.4)$ & \\
\hline \multicolumn{8}{|c|}{ rs7087507 } \\
\hline GG & $64(13.0)$ & $40(16.5)$ & 0.12 & $66(13.3)$ & 0.016 & $106(14.4)$ & 0.014 \\
\hline$A G$ & $191(38.9)$ & $105(43.2)$ & & $234(47.3)$ & & 339 (45.9) & \\
\hline $\mathrm{AA}$ & $236(48.1)$ & $98(40.0)$ & & $195(39.4)$ & & 293 (39.7) & \\
\hline$G$ & 319 (32.6) & $185(38.1)$ & 0.034 & $366(37.0)$ & 0.04 & 551 (37.3) & 0.014 \\
\hline A & $663(67.4)$ & 301 (61.9) & & $624(63.0)$ & & $925(62.7)$ & \\
\hline \multicolumn{8}{|c|}{ rs10761600 } \\
\hline $\mathrm{TT}$ & $108(21.6)$ & $30(12.4)$ & 0.01 & $71(14.2)$ & 0.009 & $101(13.6)$ & 0.001 \\
\hline AT & $221(44.2)$ & $117(48.3)$ & & $243(48.6)$ & & $360(48.5)$ & \\
\hline $\mathrm{AA}$ & $171(34.2)$ & 95 (39.3) & & $186(37.2)$ & & 281 (37.9) & \\
\hline $\mathrm{T}$ & $437(43.6)$ & $177(36.6)$ & 0.0089 & $376(61.7)$ & 0.017 & $562(37.9)$ & 0.0037 \\
\hline$A$ & $563(56.4)$ & $307(63.4)$ & & $606(38.3)$ & & $922(62.1)$ & \\
\hline
\end{tabular}

Abbreviations: ARID, AT-rich interaction domain; MRF2, modulator recognition factor-2; SNP, single-nucleotide polymorphism; T2D, type 2 diabetes.

aSignificance indicates control 1 vs T2D.

bSignificance indicates control 2 vs T2D.

cSignificance indicates controls 1 and 2 vs T2D.

Values were given as allele or genotype frequencies and proportions (\%); differences were compared by chi-square test.

$n$ refers to numbers of individuals.

$0.50-0.83), P=0007)$. For the SNP of rs10761600, compared with codominant TT, AT and AA decreased risk of T2D by 42 and $43 \%$, and AA or AT(dominant model) prevented individual from being T2D than TT (OR 0.58 (95\% CI 0.42-0.78), $P=0.00039$ ). Regarding SNPs rs7901348, rs2278308, rs10821944 and rs12357548, no additional associations were found.

\section{LD between MRF2/ARID5B SNPs and T2D}

A total of 10 haplotype blocks were defined, as indicated using Japanese data from the HapMap database (Figure 1). Strong LD was detected among rs2893880, rs10740055, rs7087507 and rs10761600, which were located within the third haplotype block. rs7901348, rs2278308, rs10821944 and rs12357548 were not included in this block. Five major haplotype combinations with prevalence of $>5 \%$ are shown in Table 4. Combination of CAAA was the most prevalent haplotype (28.2 and $29.9 \%$ for the diabetes and the combined control group, respectively). Haplotype analysis revealed that the second prominent haplotype CAAT was significantly more prevalent in the T2D group (26.5 vs $17.2 \%$ ), and the haplotype GCGA was more prevalent in the combined control group $(21.4$ vs $18.0 \%)(P<0.001$ and $P=0.038$, respectively). No difference was observed between the two control groups.

Haplotypes other than GCGA showed an increased risk for diabetes with OR 1.24 (95\% CI 1.01-1.53) (Figure 2). Individuals with the combination of CAAT alleles indicated an $84 \%$ increased prevalence of diabetes compared to individuals with GCGA (OR 1.84 (95\% CI 1.43-2.37)). The significance was remained after adjusting for age, gender and BMI (OR 1.86 (95\% CI 1.43-2.41)).

\section{Associations of MRF2/ARID5B with plasma adiponectin levels and other clinical factors}

The associations of MRF2/ARID5B SNPs with adiponectin level and other clinical factors were explored where data was available. The levels of clinical factors were compared between individuals with haplotypes CAAT and haplotype other than CAAT (others). A multivariable analysis (multivariable analysis 1, Table 5) adjusting for age, gender and BMI indicated that higher $\mathrm{HbAlc}$ level $(P<0.001)$, higher FPG level $(P=0.036)$ and lower adiponectin level $(P=0.028)$ were associated with higher frequency of haplotype CAAT.

Because there were moderate correlations of BMI with leptin, HOMA-IR and HOMA- $\beta$ ( $r=0.47,0.45$ and 0.38 , respectively), a multivariable analysis adjusting for only age and gender (multivariable analysis 2 , Table 5) was performed to avoid multicollinearity. In addition to HbAlc, FPG and adiponectin, leptin $(P=0.003)$ and HOMA-IR $(P=0.024)$ showed a significant association with haplotype CAAT. No significant association was observed for BMI, insulin resistance index, or HOMA- $\beta$ within this study population. 
Table 3 Association between MRF2/ARID5B SNPs and T2D with multivariable regression

\begin{tabular}{|c|c|c|c|c|c|c|}
\hline & \multicolumn{2}{|c|}{ T2D vs control 1 ( $\mathrm{n}=500$ vs 243 ) } & \multicolumn{2}{|c|}{ T2D vs control $2(\mathrm{n}=500$ vs 500$)$} & \multicolumn{2}{|c|}{ T2D vs control 1 and 2 ( $n=500$ vs 743) } \\
\hline \multicolumn{7}{|l|}{ Co-dominant } \\
\hline GG & ref. & & ref. & & ref. & \\
\hline CG & $1.42(0.81-2.48)$ & 0.22 & $1.26(0.81-1.96)$ & 0.3 & $1.27(0.86-1.87)$ & 0.22 \\
\hline $\mathrm{CC}$ & $1.60(0.92-2.78)$ & 0.093 & $1.56(1.01-2.43)$ & 0.046 & $1.52(1.04-2.23)$ & 0.032 \\
\hline \multicolumn{7}{|l|}{ Recessive } \\
\hline $\mathrm{GG}+\mathrm{CG} / \mathrm{CC}$ & $0.81(0.57-1.16)$ & 0.26 & $0.77(0.59-1.00)$ & 0.054 & $0.79(0.63-1.00)$ & 0.051 \\
\hline \multicolumn{7}{|l|}{ rs10740055 } \\
\hline \multicolumn{7}{|l|}{ Co-dominant } \\
\hline CC & ref. & & ref. & & ref. & \\
\hline $\mathrm{CC}+\mathrm{AC} / \mathrm{AA}$ & $0.61(0.41-0.09)$ & 0.014 & $0.64(0.47-0.86)$ & 0.003 & $0.64(0.50-0.83)$ & 0.0007 \\
\hline \multicolumn{7}{|l|}{ rs7087507 } \\
\hline \multicolumn{7}{|l|}{ Co-dominant } \\
\hline GG & ref. & & ref. & & ref. & \\
\hline$A G$ & $1.03(0.60-1.75)$ & 0.92 & $0.91(0.60-1.38)$ & 0.66 & $0.94(0.66-1.36)$ & 0.76 \\
\hline AA & $1.42(0.84-2.42)$ & 0.192 & $1.33(0.87-2.02)$ & 0.19 & $1.34(0.93-1.93)$ & 0.11 \\
\hline \multicolumn{7}{|l|}{ Dominant } \\
\hline$A A+A G / G G$ & $1.22(0.74-2.00)$ & 0.43 & $1.10(0.74-1.63)$ & 0.64 & $1.13(0.80-1.59)$ & 0.49 \\
\hline \multicolumn{7}{|l|}{ Recessive } \\
\hline$G G+A G / A A$ & $0.72(0.50-1.02)$ & 0.066 & $0.70(0.53-0.92)$ & 0.01 & $0.71(0.56-0.90)$ & 0.005 \\
\hline \multicolumn{7}{|l|}{ rs10761600 } \\
\hline
\end{tabular}

Abbreviations: ARID, AT-rich interaction domain; Cl, confidence interval; MRF2, modulator recognition factor-2; OR, odds ratio; SNP, single nucleotide polymorphism; T2D, type 2 diabetes. ORs were adjusted for age, sex and body mass index.

Table 4 Haplotype analysis for MRF2/ARID5B SNPs and T2D

\begin{tabular}{lcccc}
\hline \multicolumn{5}{c}{ Controls 1 and } \\
Haplotype & Frequency (\%) & Frequency (\%) & Frequency (\%) & $\mathrm{P}^{b}$ \\
\hline CAAA & $273(28.2)$ & $437(29.9)$ & $710(29.2)$ & 0.37 \\
CAAT & $257(26.5)$ & $251(17.2)$ & $508(20.9)$ & $<0.001$ \\
GCGA & $174(18.0)$ & $313(21.4)$ & $487(20.0)$ & 0.038 \\
GCGT & $106(11.0)$ & $178(12.2)$ & $284(11.7)$ & 0.358 \\
CCAA & $66(6.8)$ & $108(7.4)$ & $174(7.2)$ & 0.594
\end{tabular}

Abbreviations: ARID, AT-rich interaction domain; MRF2, modulator recognition factor-2; SNP, single-nucleotide polymorphism; T2D, type 2 diabetes.

aTotal indicates the combining of T2D patients and control individuals.

bSignificance represents comparison of each haplotype type with all other haplotype combination in the T2D and that of the controls 1 and 2 group.

Data depicted five major haplotype combinations with frequency $>5 \%$.

Values were given as haplotype frequencies and proportions (\%) of the total combinations, and compared with chi-square test. $n$ refers to numbers of individuals.

\section{DISCUSSION}

To our knowledge, this is the first study in humans investigating the relation of MRF2/ARID5B variations with the presence of T2D. We found that variants of rs2893880, rs10740055, rs7087507 and rs10761600 on the MRF2/ARID5B gene were associated with T2D; carrying $\mathrm{C}, \mathrm{A}, \mathrm{A}$ and $\mathrm{T}$ seemed to increase the risk of T2D, and was associated with higher HbAlc and FPG level. CAAT was also related to lower adiponectin level and higher leptin level. These findings are concordant with our previous study involving CAD patients. Although the associations of MRF2/ARID5B SNPs with diabetes and its associations with CAD may be independent from each other, it is considered that the former may be, at least, partially responsible for the latter.

Variations involved in our studies were located within the second and third intron regions of MRF2/ARID5Bbeta with an open reading frame of $3564 \mathrm{bp}$, and no coding SNPs were observed in exon 3 or exon 4 as indicated by Wanget al. ${ }^{15}$ and Trevino et al.; ${ }^{24}$ therefore, these intronic polymorphisms are much more likely to have a modifying effect by influencing the expression level of MRF2/ARID5B by 
themselves or by other unknown causative genetic variations located within or nearby the haplotype block that remains to be elucidated.

Regarding the mechanisms through which MRF2/ARID5B SNPs are involved in the presence of diabetes, several possibilities could be considered. First, based on the finding from mice lacking the Mrf2/ Arid5b gene, ${ }^{9-11}$ and the in vivo and in vitro evidence provided by Donget al. ${ }^{12}$ and Yamakawa et al., ${ }^{13,14}$ MRF2/ARID5B may influence diabetes through its regulation of adipogenesis. Second, gene targeting of Mrf2/Arid5b also showed the homozygous mutant presented reduced viability and displayed male and female reproductive organ abnormalities and adrenal gland abnormalities, ${ }^{9,10}$ suggesting that Mrf2/Arid5b likely has important roles in the endocrine system and influences insulin resistance. We revealed that MRF2/ARID5B seemed to be associated with HOMA-IR, which may support this hypothesis. In addition, MRF2/ARID5B was cloned as a key regulator for smooth muscle cell differentiation, ${ }^{8}$ suggesting that the mechanism involved in the associations of MRF2/ARID5B SNPs with CAD may be also responsible for its association with diabetes. Finally, adiponectin is a

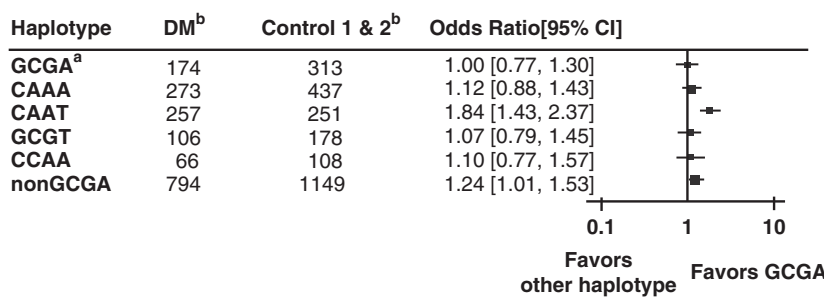

Figure 2 The association of other haplotypes with type 2 diabetes (T2D) in comparison to GCGA. ${ }^{a}$ Reference haplotype; ${ }^{b}$ data were given as number of each of the five major haplotypes. Non-GCGA indicates all haplotypes other than GCGA. Odds ratio $(O R)=$ odds of T2D in subjects with each of the other haplotypes/odds of T2D in subjects with GCGA. Box represents each $\mathrm{OR}$. The size of each box is proportional to the inverse variance weight of the estimated effect size. Horizontal lines represent 95\% confidence interval $(\mathrm{Cl})$ of $\mathrm{OR}$. The vertical line is at the null OR. well-known antidiabetic adipocytokine ${ }^{25}$ and believed to have crucial roles in the regulation of energy homeostasis and insulin sensitivity. ${ }^{26}$ Our findings in the present study support speculation that the MRF2/ $A R I D 5 B$ variations may be involved in the pathogenesis of diabetes through regulating the adiponectin level. Though we do not have any data yet to reveal the mechanisms, we could speculate it from the analysis of the human adiponection promoter. In the basal promoter region of human adiponectin, PPRE, SREBP and C/EBP are important for transcriptional activation. ${ }^{27,28}$ As MRF2/ARID5B protein was reported to recognize a five-base core sequence (AATA $(\mathrm{C} / \mathrm{T}))$, we found several possible MRF2 binding sites in this region. ${ }^{7}$ Especially, we are very interested in the A/T-rich region, which is adjacent to PPRE binding sites (from -286 to -267 ). It is possible that MRF2 may bind this region and PPARG and activate the adiponectin transcription, but further investigation is necessary.

Recently, two independent, large genome-wide association studies ${ }^{24,29}$ reported linkage of acute lymphoblastic leukemia to a locus in the $A R I D 5 B$. Several replication analysis validated the findings in different ethnic populations, ${ }^{30-32}$ including the first genome-wide association study in an Asian population. ${ }^{33}$ These studies involved variations of rs7073837, rs10994982, rs10821963, rs7896246 and rs7089424, all of which were located within the same third haplotype block as we focused on. Besides, a newly reported meta-analysis of genome-wide association study identified $A R I D 5 B$ as one of nine novel loci associated with rheumatoid in the Japanese population that was followed by a subgroup analysis identifying the association of ARID5B with Graves's disease. ${ }^{34}$ Based on these findings, MRF2/ARID5B was indicated to involve in inflammation and autoimmune. Although none of these studies investigated the associations of their SNPs with T2D or obesity, these findings could serve as a resource for replication and offer room for deeper study to identify possible biological pathways, including inflammation and autoimmune that may contribute to diabetes as well as other human disorders.

This study has some limitations. First, differences were detected as compared with a hospital-based control group and a community-

Table 5 Associations between MRF2/ARID5B haplotype CAAT and clinical factors

\begin{tabular}{|c|c|c|c|c|c|c|c|c|c|}
\hline & \multicolumn{2}{|c|}{ Haplotype } & \multirow{2}{*}{$\begin{array}{c}\text { Median } \\
\text { (25th, 75th percentiles) }\end{array}$} & \multicolumn{2}{|c|}{ Univarible analysis } & \multicolumn{2}{|c|}{ Multivarible analysis 1} & \multicolumn{2}{|c|}{ Multivarible analysis 2} \\
\hline & Type & Frequency & & OR $(95 \% \mathrm{Cl})$ & $\mathrm{P}$ & OR $(95 \% \mathrm{Cl})$ & $\mathrm{P}$ & OR $(95 \% \mathrm{Cl})$ & $\mathrm{P}$ \\
\hline \multirow[t]{2}{*}{ BMI } & CAAT & 1508 & $24.4(22.0,27.3)$ & $1.024(0.998-1.051)$ & 0.074 & ND. & ND & $1.021(0.994-1.048)$ & 0.13 \\
\hline & Others & 420 & $24.2(21.6,27)$ & & & & & & \\
\hline \multirow[t]{2}{*}{$\mathrm{HbAlc}$} & CAAT & 1514 & $6.7(5.6,8.3)$ & $1.136(1.071-1.197)$ & $<0.001$ & $1.127(1.061-1.198)$ & $<0.001$ & $1.126(1.060-1.196)$ & $<0.001$ \\
\hline & Others & 424 & $5.9(5.6,6.1)$ & & & & & & \\
\hline \multirow[t]{2}{*}{ FPG } & CAAT & 1507 & $116(96,162)$ & $1.003(1.001-1.005)$ & 0.014 & 1.002 (1.000-1.005) & 0.036 & $1.002(1.000-1.005)$ & 0.04 \\
\hline & Others & 421 & $102(91,149)$ & & & & & & \\
\hline \multirow[t]{2}{*}{ Adiponectin } & CAAT & 773 & $9.7(5.6,15.3)$ & 0.976 (0.959-0.993) & 0.007 & 0.978(0.959-0.998) & 0.028 & 0.977 (0.959-0.995) & 0.014 \\
\hline & Others & 187 & $12.1(6.3,20.1)$ & & & & & & \\
\hline \multirow[t]{2}{*}{ Leptin } & CAAT & 853 & $63(38,106)$ & $1.003(1.001-1.006)$ & 0.013 & 1.003(0.999-1.007) & 0.12 & $1.004(1.002-1.008)$ & 0.003 \\
\hline & Others & 81 & $53(29,87)$ & & & & & & \\
\hline \multirow[t]{2}{*}{ IRI } & CAAT & 1214 & $6.3(4.0,9.0)$ & 0.998 (0.979-1.017) & 0.81 & $0.990(0.968-1.012)$ & 0.37 & 0.998 (0.978-1.017) & 0.8 \\
\hline & Others & 330 & $6.4(4.8,9.0)$ & & & & & & \\
\hline \multirow[t]{2}{*}{ HOMA-IR } & CAAT & 811 & $1.59(1.18,2.37)$ & $1.147(1.019-1.291)$ & 0.023 & $1.078(0.941-1.234)$ & 0.28 & $1.146(1.018-1.290)$ & 0.024 \\
\hline & Others & 167 & $1.52(1.09,2.11)$ & & & & & & \\
\hline \multirow[t]{2}{*}{ HOMA- $\beta$} & CAAT & 811 & $85.2(66.6,116.5)$ & $1.002(0.999-1.004)$ & 0.142 & $1.001(0.998-1.003)$ & 0.63 & $1.002(0.999-1.004)$ & 0.14 \\
\hline & Others & 167 & $88.8(66.0,112.5)$ & & & & & & \\
\hline
\end{tabular}

Abbreviations: ARID, AT-rich interaction domain; BMI, body mass index; $\mathrm{Cl}$, confidence interval; FPG, fasting plasma glucose; HbA1c, hemoglobin Alc; HOMA-IR, homeostasis model assessment of insulin resistance; IRI, insulin resistance index; MRF2, modulator recognition factor-2; OR, odds ratio; T2D, type 2 diabetes.

Clinical data were presented as median (25th, 75 th percentiles).

ND indicates not determined.

Multivariable analysis 1 was adjusted for age, gender and BMI, and multivariable analysis 2 was adjusted for age and gender. 
based control group, given that these participants were included around year 2000, control group 1 involved some subjects whose HbAlc (NGSP\%) is $>6.2 \%$, and some whose age is $<40$ years, it may raise a possibility that some potential diabetes patients might be included. However, finding from these two groups were consistent with each other, and no difference were observed between these two groups in terms of MRF2/ARID5B allele and genotypic frequencies. Besides, involving hospital-based control group might possibly lead to an underestimated result; however, it is more likely to represent the real setting of general clinical practice. Second, although all disease associated variations localized within the same LD block, we could not exclude the possibility that SNPs located in other haplotype blocks, which were not investigated in this study, may have stronger association with T2D and relevant clinical factors. Besides, the SNPs assessed here are not causal variants, further function support analysis is warranted to elucidate the underlying mechanisms of them.

In summary, the findings from this study and those from our previous research implied MRF2/ARID5B as a novel susceptibility factor for both T2D and CAD. It may influence the presence of T2D through obesity or insulin resistance or both. Elucidation of the mechanisms through which the no-coding variants of the MRF2/ $A R I D 5 B$ gene are involved in susceptibility to T2D, CAD and other diseases could contribute to the further understanding of the pathogenesis of these human disorders.

\section{CONFLICT OF INTEREST}

The authors declare no conflict of interest.

\section{ACKNOWLEDGEMENTS}

This study was supported in part by a Grant-in-Aid from the Ministry of Education, Culture, Sports, Science and Technology (MEXT), Japan (to RN), and a research grant from the National Institute of Biomedical Innovation, Japan (to RN); The study was also supported in part by Special Coordination Funds for Promoting Science and Technology by MEXT, Japan (to TY and RN) and the Japan Society for the Promotion of Science (JSPS) through its Funding Program for World-Leading Innovative R\&D on Science and Technology (FIRST Program). We thank Yuki Itoh, Eri Fujiu, Chika Masuo, Yasuko Kubono and Yukino Sato for their technical assistance. We also thank Robert E Brandt (Founder and CEO, MedEd, Japan) for editing the manuscript.

1 Florez, J. C. Clinical review: the genetics of type 2 diabetes: a realistic appraisal in 2008. J. Clin. Endocrinol. Metab. 93, 4633-4642 (2008)

2 Megia, A., Gallart, L., Fernandez-Real, J. M., Vendrell, J., Simon, I., Gutierrez, C. et al. Mannose-binding lectin gene polymorphisms are associated with gestational diabetes mellitus. J. Clin. Endocrinol. Metab. 89, 5081-5087 (2004).

3 Florez, J. C., Manning, A. K., Dupuis, J., McAteer, J., Irenze, K., Gianniny, L. et al. A $100 \mathrm{~K}$ genome-wide association scan for diabetes and related traits in the Framingham Heart Study: replication and integration with other genome-wide datasets. Diabetes 56, 3063-3074 (2007).

4 Yamauchi, T., Hara, K. Maeda, S., Yasuda, K., Takahashi, A., Horikoshi, M. et al. A genome-wide association study in the Japanese population identifies susceptibility loci for type 2 diabetes at UBE2E2 and C2CD4A-C2CD4B. Nat. Genet. 42, 864-868 (2010).

5 Stumvoll, M., Goldstein, B. J. \& van Haeften, T. W. Type 2 diabetes: principles of pathogenesis and therapy. Lancet 365, 1333-1346 (2005).

6 Huang, T. H., Oka, T., Asai, T., Okada, T., Merrills, B. W., Gertson, P. N. et al. Repression by a differentiation-specific factor of the human cytomegalovirus enhancer. Nucleic Acids Res. 24, 1695-1701 (1996).

7 Whitson, R. H., Huang, T. \& Itakura, K. The novel Mrf-2 DNA-binding domain recognizes a five-base core sequence through major and minor-groove contacts. Biochem. Biophys. Res. Commun 258, 326-331 (1999).

8 Watanabe, M., Layne, M. D., Hsieh, C. M., Maemura, K., Gray, S., Lee, M. E. et al. Regulation of smooth muscle cell differentiation by AT-rich interaction domain transcription factors Mrf2alpha and Mrf2beta. Circ. Res. 91, 382-389 (2002).
9 Ristevski, S., Tam, P. P., Kola, I. \& Hertzog, P. Desrt an AT-rich interaction domain family transcription factor gene, is an early marker for nephrogenic mesoderm and is expressed dynamically during mouse limb development. Mech. Dev. 104, 139-142 (2001).

10 Lahoud, M. H., Ristevski, S., Venter, D. J., Jermiin, L. S., Bertoncello, I., Zavarsek, S. et al. Gene targeting of Desrt, a novel ARID class DNA-binding protein, causes growth retardation and abnormal development of reproductive organs. Genome Res. 11, 1327-1334 (2001).

11 Whitson, R. H., Tsark, W., Huang, T. H. \& Itakura, K. Neonatal mortality and leanness in mice lacking the ARID transcription factor Mrf-2. Biochem. Biophys. Res. Commun. 312, 997-1004 (2003).

12 Dong, J., Ishimori, N., Paigen, B., Tsutsui, H. \& Fujii, S. Role of modulator recognition factor 2 in adipogenesis and leptin expression in 3T3-L1 cells. Biochem. Biophys. Res. Commun. 366, 551-555 (2008).

13 Yamakawa, T., Whitson, R. H., Li, S. L. \& Itakura, K. Modulator recognition factor-2 is required for adipogenesis in mouse embryo fibroblasts and 3T3-L1 cells. Mol. Endocrinol. 22, 441-453 (2008).

14 Yamakawa, T., Sugimoto, K., Whitson, R. H. \& Itakura, K. Modulator recognition factor2 regulates triglyceride metabolism in adipocytes. Biochem. Biophys. Res. Commun. 391, 277-281 (2010).

15 Wang, G., Watanabe, M., Imai, Y., Hara, K., Manabe, I., Maemura, K. et al. Genetic variations of Mrf-2/ARID5B confer risk of coronary atherosclerosis in the Japanese population. Int. Heart J. 49, 313-327 (2008).

16 Hara, K., Boutin, P., Mori, Y., Tobe, K., Dina, C., Yasuda, K. et al. Genetic variation in the gene encoding adiponectin is associated with an increased risk of type 2 diabetes in the Japanese population. Diabetes 51, 536-540 (2002).

17 Horikoshi, M., Hara, K., Ohashi, J., Miyake, K., Tokunaga, K., Ito, C. et al. A polymorphism in the AMPKalpha2 subunit gene is associated with insulin resistance and type 2 diabetes in the Japanese population. Diabetes 55, 919-923 (2006).

18 Kashiwagi, A., Kasuga, M., Araki, E., Oka, Y., Hanafusa, T., Ito, H. et al. International clinical harmonization of glycated hemoglobin in Japan: From Japan diabetes society to national glycohemoglobin standardization program values. J. Diabetes Invest. 3, 39-40 (2012)

19 Tanita, T., Miyakoshi, H. \& Nakano, Y. Performance of ELISA for specific measurement of high-molecular-weight (HMW) adiponectin. J. Immunol. Methods 333, 139-146 (2008).

20 Matthews, D. R., Hosker, J. P., Rudenski, A. S., Naylor, B. A., Treacher, D. F. \& Turner, R. C. Homeostasis model assessment: insulin resistance and beta-cell function from fasting plasma glucose and insulin concentrations in man. Diabetologia 28, 412-419 (1985).

21 Nakai, K., Habano, W., Fujita, T., Schnackenberg, J., Kawazoe, K., Suwabe, A. et al. Highly multiplexed genotyping of coronary artery disease-associated SNPs using MALDI-TOF mass spectrometry. Hum. Mutat. 20, 133-138 (2002).

22 Gabriel, S. B., Schaffner, S. F., Nguyen, H., Moore, J. M., Roy, J., Blumenstiel, B. et al. The structure of haplotype blocks in the human genome. Science 296, 2225-2229 (2002).

23 Purcell, S., Daly, M. J. \& Sham, P. C. WHAP: haplotype-based association analysis. Bioinformatics 23, 255-256 (2007)

24 Trevino, L. R., Yang, W., French, D., Hunger, S. P., Carroll, W. L., Devidas, M. et al. Germline genomic variants associated with childhood acute lymphoblastic leukemia. Nat. Genet. 41, 1001-1005 (2009).

25 Kadowaki, T. \& Yamauchi, T. Adiponectin and adiponectin receptors. Endocr. Rev. 26, 439-451 (2005).

26 Yamauchi, T., Nio, Y., Maki, T., Kobayashi, M., Takazawa, T., Iwabu, M. et al. Targeted disruption of AdipoR1 and AdipoR2 causes abrogation of adiponectin binding and metabolic actions. Nat. Med. 13, 332-339 (2007).

$27 \mathrm{Liu}$, M. \& Liu, F. Transcriptional and post-translational regulation of adiponectin. Biochem. J. 425, 41-52 (2010).

28 Iwaki, M., Matsuda, M., Maeda, N., Funahashi, T., Matsuzawa, Y., Makishima, M. et al. Induction of adiponectin, a fat-derived antidiabetic and antiatherogenic factor, by nuclear receptors. Diabetes 52, 1655-1663 (2003).

29 Papaemmanuil, E., Hosking, F. J., Vijayakrishnan, J., Price, A., Olver, B., Sheridan, E. et al. Loci on 7p12.2, 10q21.2 and 14q11.2 are associated with risk of childhood acute lymphoblastic leukemia. Nat. Genet. 41, 1006-1010 (2009).

30 Yang, W., Trevino, L. R., Yang, J. J., Scheet, P., Pui, C. H., Evans, W. E. et al. ARID5B SNP rs10821936 is associated with risk of childhood acute lymphoblastic leukemia in blacks and contributes to racial differences in leukemia incidence. Leukemia 24, 894-896 (2010).

31 Healy, J., Richer, C., Bourgey, M., Kritikou, E. A. \& Sinnett, D. Replication analysis confirms the association of ARID5B with childhood B-cell acute lymphoblastic leukemia. Haematologica 95, 1608-1611 (2010).

32 Prasad, R. B., Hosking, F. J., Vijayakrishnan, J., Papaemmanuil, E., Koehler, R., Greaves, M. et al. Verification of the susceptibility loci on 7p12.2, 10q21.2, and $14 q 11.2$ in precursor B-cell acute lymphoblastic leukemia of childhood. Blood 115, 1765-1767 (2010).

33 Han, S., Lee, K. M., Park, S. K., Lee, J. E., Ahn, H. S., Shin, H. Y. et al. Genome-wide association study of childhood acute lymphoblastic leukemia in Korea. Leuk. Res. 34, 1271-1274 (2010).

34 Okada, Y., Terao, C., Ikari, K., Kochi, Y., Ohmura, K., Suzuki, A. et al. Meta-analysis identifies nine new loci associated with rheumatoid arthritis in the Japanese population. Nat. Genet. 44, 511-516 (2012). 